\title{
A new approach to the solution of Maxwell's equations for low frequency and high-resolution biomedical problems
}

Huawei Zhao Stuart Crozier Feng Liu* (Received 14 January 2003)

\begin{abstract}
High spatial resolution studies of the interaction of the human body with electromagnetic waves of low frequency presents a difficult computational problem. As these studies typically require at least $10^{4}$ points per wavelength, a huge number of time steps would be needed to be able to use the finite difference time domain method (FDTD). In this paper, a new technique is described, which allows the FDTD method to be efficiently applied over a very large frequency range, including low frequencies. In the method, no alterations to the properties of either the source or the transmission media are required. The method is essentially frequency independent and has been verified against analytical solutions within the
\end{abstract}

*Centre for Magnetic Resonance, The University of Queensland, St. Lucia, Brisbane, Queensland 4072, Australia. mailto: zhao@cmr.uq. edu. au

${ }^{0}$ See http://anziamj . austms.org.au/V44/CTAC2001/Zhao for this article, (C) Austral. Mathematical Soc. 2003. Published 1 April 2003. ISSN 1446-8735 
frequency range 50 Hertz to 1 Gigahertz. As an example of the lower frequency range, the method has been applied to the simulation of electromagnetic field behavior in the human body exposed to the pulsed magnetic field gradients of a magnetic resonance image (MRI) system.

\section{Contents}

1 Introduction

C852

2 The HR-FDTD method

C855

3 Verification

C859

4 Modeling the gradient coil in MRI system

C862

5 Simulation of the human body in an MR scanner C864

6 Conclusion

C866

References

C866

\section{Introduction}

The applications of electromagnetic (EM) radiation are everywhere in the modern world, from personal wireless communication services (for example, cellular phones, paging systems, wireless Internet devices, etc), microwave heating processes, military systems and medicine. Since A'Arsonval's first use of magnetic fields to treat a number of medical conditions in the late 19th century, there has 
been a fascination with the interaction of these fields with biological systems and the human body $[2,6]$. Today, there are numerous and diverse applications of time-varying magnetic fields in medical research and clinical practice. With the current surge of research and development in the field of Bioelectromagnetics (BioEM), many applications remain in their infancy and, in many cases, both the scientific and clinical evidence of their effects is questionable.

Since 1982, magnetic resonance imaging (MRI) has been available clinically with the first publication of the nuclear magnetic resonance (NMR) images of the human body. NMR imaging provides superior detection capabilities of abnormal tissue structures occurring in the brain, spinal cord, various abdominal regions, the breast, and the cardiovascular and musculoskeletal system. As scientists and engineers develop MR designs with higher field strengths and faster scanning, the complexity of the interaction between the EM field and the human body increases [7].

In an MRI, short and intense current pulses (that is, within the micro- to millisecond range) are supplied to one or more coils to produce rapidly switched strong magnetic fields. These fields may have the unfortunate side effect of stimulating nerve fibers in the cerebral cortex or in the peripheral nerves of the test subject. The pulses are repeated at low rates of tens per second. MRI and MRS involve two types of time-varying fields: a slowly time-varying non-uniform field (that is, of a frequency below $3 \mathrm{~T} / \mathrm{s}$ ) and a radio frequency $(\mathrm{RF})$ field (that is, in the megahertz frequency range) with moderate intensities.

In most cases it is almost impossible to perform experimentation to study the interactions between EM fields and the human body on both physical and ethical grounds. In instrument design, an MR engineer concentrates on physical performance, cost minimization and efficiency, with little consideration of the effects of 
human interaction with the EM field. In order to completely quantify the interaction of EM field interactions with the human body, a complete description of the EM field at every point in the system, including at every point of the human body, is necessary for the further development of MR technology. This kind of detail cannot be derived experimentally and the only way that this can be approximated is through the use of a sophisticated three-dimensional computational model.

The finite difference time domain (FDTD) technique is a well established numerical technique for computationally modeling EM problems. It is applicable for very complicated, inhomogeneous dielectric structures. The technique was first proposed by Yee [9] and later developed by Umashankar and Taflove [8]. Recently, there has been increased research in applying the FDTD technique to obtain a high-resolution (HR) (in the order of points per wavelength) solution for a diverse range of BioEm problems [3, 5]. However, current applications of the conventional FDTD technique can be hampered by the intrinsic time stepping stability criterion $(\Delta t \leq$ $1 /\left\{c \sqrt{1 / \Delta x^{2}+1 / \Delta y^{2}+1 / \Delta z^{2}}\right\}$ ), where $c$ is the speed of light. If the required resolution is in the order of $10^{2} \mathrm{~m}$ or less and the wavelength is in the order of $10^{3} \mathrm{~m}$, the conventional application of the FDTD technique results in a high definition problem that is notoriously difficult to solve due to the significant computation expense required.

For example, to obtain the steady state sinusoidal FDTD technique solution for microwaves in the conventional manner, computational times in the order of several periods of the source are needed. In this case, approximately ten points per wavelength would be used and for high definition problems, the resolution required is in the order of $10^{4}$ points per wavelength. If a frequency of $100 \mathrm{~Hz}$ (or equivalently a wavelength of $3 \times 10^{6} \mathrm{~m}$ in free space) with a spatial 
discretization of $\Delta x=\Delta y=\Delta z=0.01 \mathrm{~m}$ is required, the intrinsic time stepping stability criterion dictates that the duration of one time step would be $\Delta t \leq \Delta x /(\sqrt{3} c)=1.9 \times 10^{-11} \mathrm{~s}$. Thus, for only a single period of the source field, the number of time steps required for the simulation would be approximately $5.3 \times 10^{8}$. To apply this approach to the study of the interactions between EM fields and the human body would result in a computational time in the order of one hundred years (with current computational technology).

Hence, the challenge for the FDTD technique is to meet practical computational requirements and deliver a HR solution for the computational model. In the past, various approximations have been used so that FDTD methods could operate at low frequencies $[3,5,4,2]$. For the complex system of the electromagnetic fields generated by an MRI system interacting with the human body, it is advantageous to run the model at a variety of frequencies without changing any of the physical properties of the system. Specifically, the induced fields due to pulsed gradient coils are in the low frequency regime and, at the other extreme, the radio frequency fields used in MRI may be up to $340 \mathrm{MHz}$. Naturally, the dielectric and conductive properties of tissue change markedly with frequency and our model of the human body has frequency dependent parameters for each tissue type. We have therefore developed a new model for these applications in which frequency scaling, dielectric constant alteration, or modification of the input source have not been used.

\section{The HR-FDTD method}

The Faraday and Ampere Laws provide a system of equations that form an independent set of coupled relationships between the timevarying electric field and magnetic field quantities. The time-varying 
EM field is described by Maxwell's equation in their vector form:

$$
\frac{\partial \mathbf{F}}{\partial t}+\nabla \times \mathbf{G}=\mathbf{I},
$$

where, with parentheses denoting a column vector, $\mathbf{F}=(\mathbf{D}, \mathbf{B})$, $\mathbf{G}=(-\mathbf{H}, \mathbf{E})$, and $\mathbf{I}=(-\mathbf{J}, \mathbf{0})$. $\mathbf{D}$ is the electric displacement, $\mathbf{B}$ is the magnetic flux density, $\mathbf{E}$ is the electric field intensity, $\mathbf{H}$ is the magnetic field intensity, and $\mathbf{J}$ is the current density. The constitutive relation is $\mathbf{F}=\mathbf{a} \cdot \mathbf{G}$ where

$$
\mathbf{a}=\left[\begin{array}{cc}
0 & \epsilon \\
-\mu & 0
\end{array}\right],
$$

$\epsilon$ is the permittivity and $\mu$ is the permeability. The electric current density $\mathbf{J}=\sigma \mathbf{E}$, and $\sigma$ is the conductivity.

In general, a field in the solution space of equation (1) can be represented as

$$
f(\mathbf{r}, t)=\sum_{i=1}^{n} A_{i}(\mathbf{r}) \sin \left(\omega_{i} t+\phi_{i}(\mathbf{r})\right),
$$

where $A$ is the amplitude of the field, $\phi$ is the phase, $\mathbf{r}$ is the position vector, and $f(\mathbf{r}, t)$ represents electric or magnetic fields. In equation (2), $\omega$ is the frequency of the source field. At any point in space, if $A_{i}$ and $\phi_{i}$ were known, then the behaviour of the full, temporal field could be described.

For high frequency problems, the time domain solution of the general field $f(\mathbf{r}, t)$ can be obtained by first applying the conventional FDTD technique and then using the appropriate Fourier transform to find the amplitude and phase terms. However, for low frequency problems, it is not feasible to run the conventional FDTD technique for a full period. Since EM field behavior is periodic as 
shown in equation (2), the full solution should be obtained immediately after the transient state. Therefore, the simulation time is only dependent on the transient response time. Furthermore, there are two types of transient behaviors, oscillations due to numerical instability and those due to the physical EM wave. Numerical oscillations are mainly caused by the rapid transition of the leading edge of the input source waveform and discontinuous interfaces within the media. These oscillations appear as high frequency contaminations of the real field and are normally present only at the wavefront. In general, these instabilities die out before the physical field reaches a steady state. Moreover, applying a "soft start" treatment [5] can significantly reduce the numerical instabilities. In this case, the transient response time is overwhelmingly dependent on the physical field. The physical field transient behavior decay is dominated by the multiple reflections of the waves. If the numerical scheme is able to instantaneously detect amplitude and phase, then, a large number of iterations are not required to obtain a solution. The number of iterations required for the simulation only depends on the time point at which the transient response decays to a stable level.

In order to instantaneously detect amplitude and phases, a different time-frequency conversion technique has to be adopted. In our proposed method, only a finite number of solutions are needed in time domain, and then an inverse approach is used to calculate $A_{i}$ and $\phi_{i}$.

Assume that the transient response will die out after $t_{t}$. If a sequence of instantaneous solutions, $\mathbf{f}=\left(f_{1}, f_{2}, \ldots, f_{m}\right)$, are recorded with respect to time $t_{1}, t_{2}, \ldots, t_{m}, t_{1} \geq t_{t}$ and $t_{i}<t_{j}$ (if $i<j$, for all $i$ and $j \in m$ ) at a point in space, then a system of non-linear equation is

$$
f_{j}(\mathbf{r}, t)=\sum_{i=1}^{n} A_{i}(\mathbf{r}) \sin \left(\omega_{i} t_{j}+\phi_{i}(\mathbf{r})\right),
$$


for $j=1,2, \ldots, m$, and $m \geq 2 n$. Expanding the functions in equation (3), and changing the variables

$$
x_{2 i-1}=A_{i} \sin \phi_{i}, \quad x_{2 i}=A_{i} \cos \phi_{i}, \quad i=1,2, \ldots, n,
$$

and

$$
\phi_{i}=\tan ^{-1}\left(\frac{x_{2 i-1}}{x_{2 i}}\right), \quad A_{i}=\sqrt{x_{2 i-1}^{2}+x_{2 i}^{2}} ;
$$

equation (4) is then written as

$$
\mathrm{Ax}=\mathbf{f}
$$

where the matrix $\mathbf{A}$ has the elements in the form of

$$
a_{i(2 j-1)}=\cos \left(\omega_{j} t_{i}\right), \quad a_{i(2 j)}=\sin \left(\omega_{j} t_{i}\right),
$$

for $i=1,2, \ldots, m, j=1,2, \ldots, n$. Since the components of the matrix are in the form of $\cos (\omega t)$ and $\sin (\omega t)$, and where $\omega t$ is very small, consequently these components are either close to one or close to zero, therefore, the matrix $\mathbf{A}$ is nearly singular. However, in BioEM problems, as the dielectric property of the human body is frequency dependent, it is better to simulate the EM separately for each individual frequency. In this case, for a single frequency source, the solution of (6) is explicitly written as

$$
\phi=\tan ^{-1}\left(\frac{\xi}{\eta}\right), \quad A=\frac{\sqrt{\xi^{2}+\eta^{2}}}{\psi},
$$

where $\xi=\cos \left(\omega t_{2}\right) f_{1}-\cos \left(\omega t_{1}\right) f_{2}, \eta=\sin \left(\omega t_{2}\right) f_{2}-\sin \left(\omega t_{2}\right) f_{1}$ and $\psi=\sin \left(\omega\left(t_{1}-t_{2}\right)\right)$. Equation (8) can be accurately solved without difficulty. 


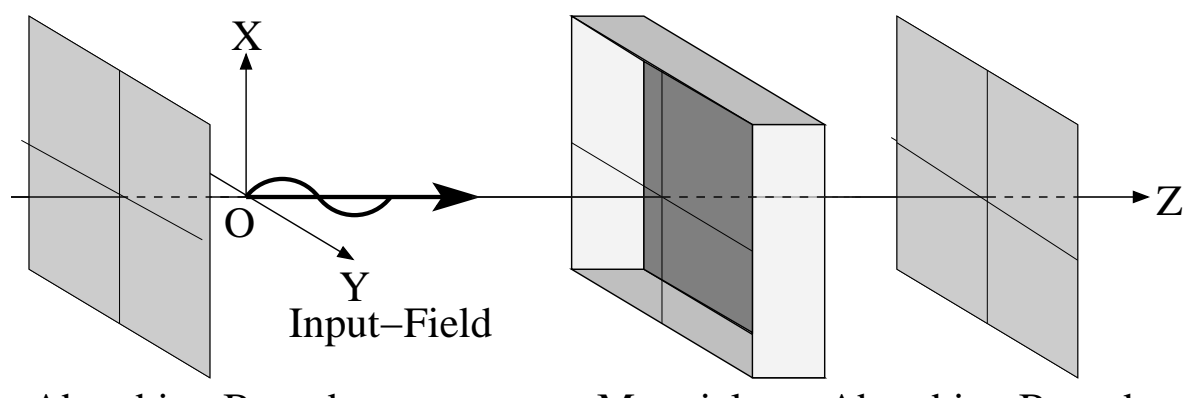

Absorbing Boundary

Material

Absorbing Boundary

Figure 1: The topology of the test model.

\section{Verification}

To verify the proposed HR-FDTD method, firstly, we describe a loop time $T_{L}$ of the field in the longest path in the system, which relates the state of convergence to the number of reflections

$$
T_{L}=\Delta t \times N_{L}=\left(2 \Delta t \times \sqrt{i_{\max }^{2}+j_{\max }^{2}+k_{\max }^{2}}\right) / C_{f l},
$$

where: $i_{\max }, j_{\max }$, and $k_{\max }$ are the maximum mesh sizes of the system, $N_{L}$ is the total number of iterations required for the signal to travel one loop, and $C_{f l}(\leq 1)$ is the stability control number.

Consider a one-dimensional plane wave problem as shown in Figure 1. This is a simple problem, for which the analytical solution is available. Berenger's PML [1] was placed on both the left and right ends to absorb the transmitted wave. Numerical simulations of electric fields were performed at two different frequencies, $1 \mathrm{GHz}$ and $50 \mathrm{~Hz}$. In all the numerical simulations, the physical time of the solutions obtained refer to $t_{2}$ with $t_{2}-t_{1}=100 \Delta t$ n.

Firstly, a $1 \mathrm{GHz}$ pure travelling wave problem was analyzed. The wavelength is $\lambda \approx 0.3 \mathrm{~m}$ and the space dimension is $1 \mathrm{~m}$ without any 
dielectric material. To test the HR-FDTD scheme, the space step size $\Delta=1.11 \mathrm{~mm}$ was used with 900 points in the solution domain. Two solutions were obtained (see Figure $2 \mathrm{a}$ and $\mathrm{b}$ ), one was recorded at 526 iterations and one at 1095 iterations. Since there is no reflection activity involved, a transient response should not be observed in the system. This behavior is confirmed by there results in both the amplitude and phase at the time of solution. A true solution is obtained as soon as the field signal reached the end of physical space (Figure 2a and b) at the loop time, which is defined as a time of a wave from its source to the end of computational domain.

In the second case, the HR-FDTD scheme is tested at $50 \mathrm{~Hz}$. The space dimension was $3.0 \mathrm{~m}$, with a $1.0 \mathrm{~m}$ lossy dielectric material, $\epsilon=80$ and $\sigma=0.5$, located in the centre of the model (see Figure 1). The space discretization size was $\Delta=1.0 \mathrm{~cm}$. According to stability criterion, for a signal travelling one complete loop, a loop time of $T_{L}=635 \Delta t$ was required. This loop time is in the order of $10^{-6}$ of the source period time. The results of the convergence history are given in Figure 2, where Figure 2c presents the amplitude and Figure 2d the phase. The solutions were calculated at 1 loop, 5 loops, 10 loops and 30 loops, in which the iteration numbers were 635, 3175, 6350 and 19, 050 respectively. Accurate solutions were obtained after 30 loops (19,050 iterations).

Note that the frequency change has effected both the amplitude and the phase of the field. This is important and demonstrates that this method should be more accurate than frequency-scaled approximations and similar methods. From the above results, see that the HR-FDTD algorithm is independent of frequency, they are consistent in both cases. In the above cases, the total computation time on a SUN Enterprise 450 was less than 1 second. 

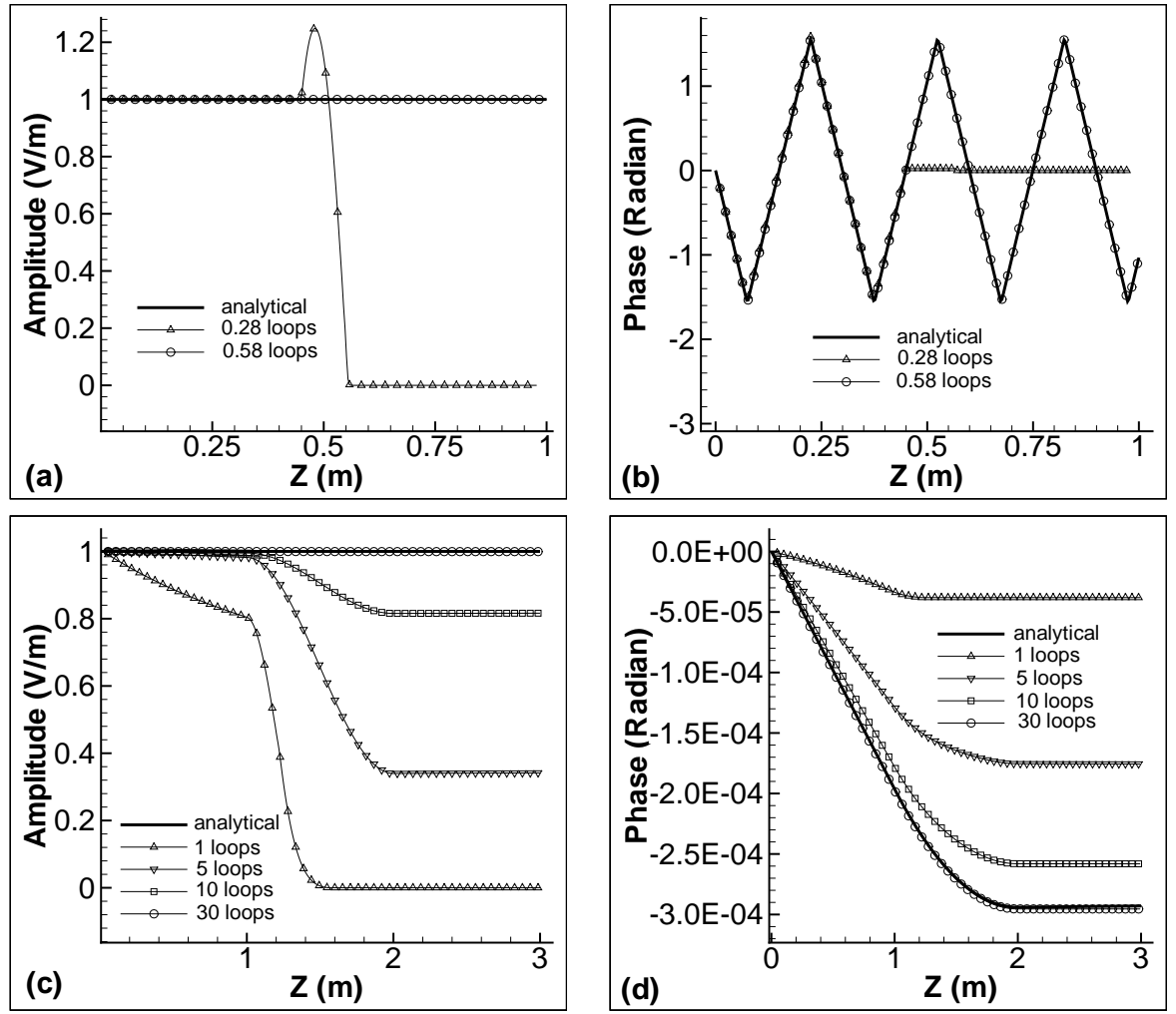

Figure 2: The calculated E-field amplitude and phase, (a) and (b) for a $1 \mathrm{GHz}$ case; (c) and (d) for a $50 \mathrm{~Hz}$ case. 


\section{Modeling the gradient coil in MRI system}

There are two types of gradient coils used in MRI, namely, the axial coil ( $Z$-coil $)$ and the transverse coils $(X$ - and $Y$-coils). In a clinical MRI system, three gradient fields work together. However, $X$-coil and $Y$-coil has the same current pattern with $\pi / 2$ rotation about $Z$-axis. In this section, the performance of the $Z$-coil and $Y$-coil are tested separately within an MRI system to demonstrate the HRFDTD method. The coil is placed in the centre of a magnet as the input current source, the magnet is treated as a perfectly conducting wall boundary, and PML (perfectly match layer) absorbing boundaries are used to truncate the two open ends of the magnet. The system was discretized using $133 \times 133 \times 260$ mesh points. The total computer memory requirement is $1.8 \mathrm{~Gb}$, and the solution was obtained after 10,000 iteration that required approximately 15 hours on a SUN Enterprise 450.

For the $Z$-coil, the typical clinical coil profiles are shown in Figure 3a. The basic magnetic field $Z$ component $\left(B_{z}\right)$ is required in a uniform distribution in the $X$ and $Y$ direction and a linear change in the $\mathrm{Z}$ direction in the centre image region. The field patterns in the $X-Z$ plane are shown in Figure 3b, which are in close agreement with Biot-Savart summations. For the $Y$-coil, the basic magnetic field should be uniform in the $Z$ and $X$ direction and change linearly in the $Y$ direction in the centre image region. The coil profile is shown in Figure 3c, which is more complex than the $Z$-coil. After HR-FDTD simulation, the final steady-state fields are presented in Figure 3d. These results highlight that the steady-state solution of the magnetic fields match the exact coil design performance. The expected behaviour of the induced electric field during the gradient switch change can be shown in details. 

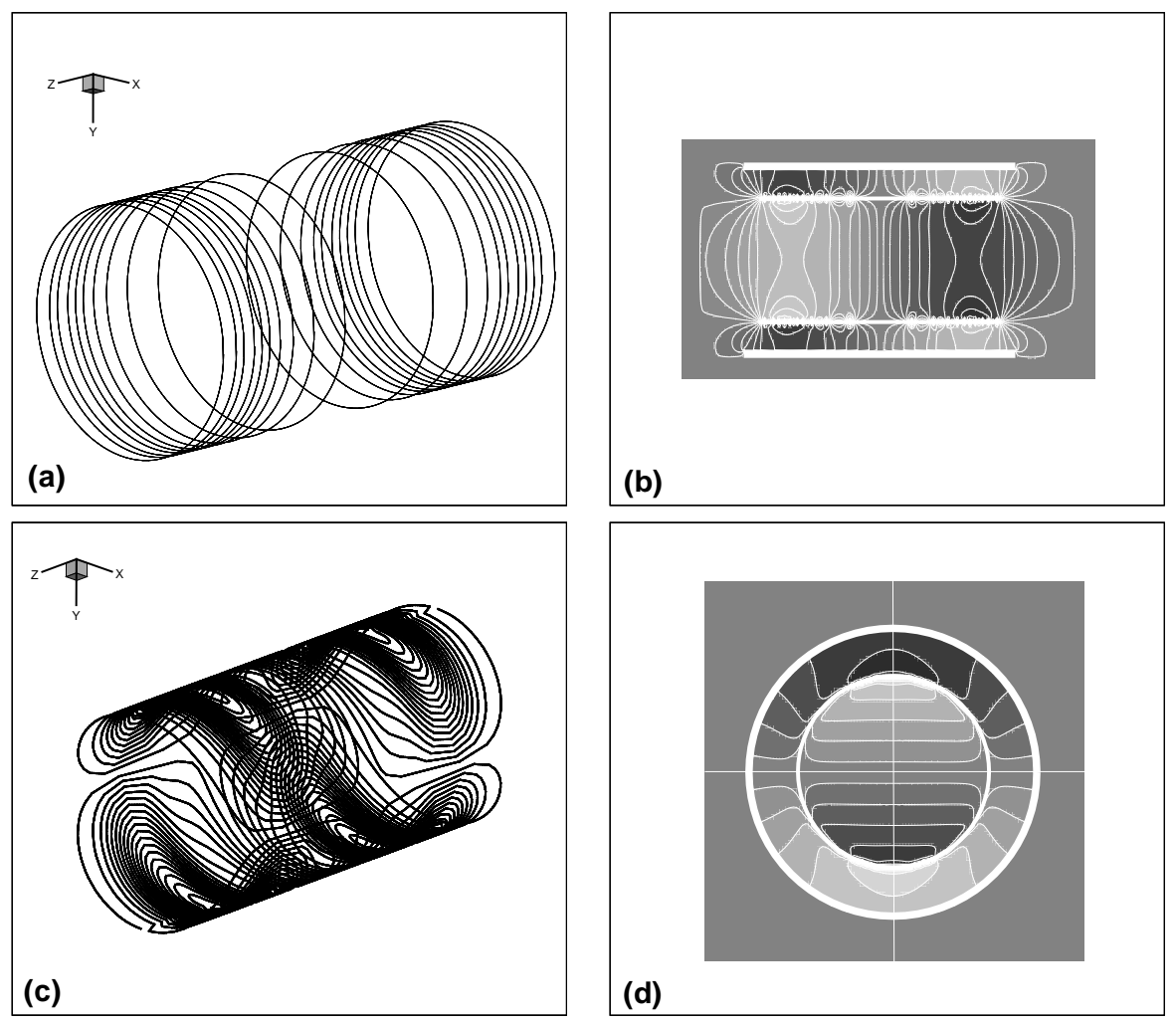

Figure 3: (a) $Z$-gradient coil profile; (b) magnetic field pattern in the centre $X-Z$ cross-section; (c) $Y$-gradient coil profile; (d) magnetic field pattern in the centre $X-Y$ cross-section. 


\section{Simulation of the human body in an MR scanner}

Gradient coils in MRI enable spatial encoding of the Magnetic Resonance signal and therefore provide the capacity to generate images. When a gradient coil is rapidly pulsed, however, eddy currents induced in the human body can be sufficient to cause peripheral nerve stimulation [7]. It is therefore important to be able to model induced currents in the body in this situation.

A human body in a magnet system with a "streamline" $Y$ gradient coil is shown in figure 4. A frequency dependent tissue model, developed by the United States Air Force Research Laboratory (http://www.brooks.af.mil/AFRL/HED/hedr), was used for these calculations. The inner surface of the magnet was treated as a perfectly conducting wall. A PML absorbing boundary, which truncates computational domain, was used to surround the human body. The current source $J_{\text {in }}=J_{0} \sin (2 \pi f \times t)$ with $f=833 \mathrm{~Hz}$ was used to drive the gradient coil. In the body model, equal space discretization was used with $\Delta x=\Delta y=\Delta z=0.8 \mathrm{~cm}$, resulting in a total of $158 \times 158 \times 276=6,890,064$ mesh points, which requires $2.6 \mathrm{~Gb}$ computer memory. In this system, one loop required about 820 iterations. After the HD-FDTD algorithm ran for 40,000 iterations, the eddy current density was obtained according to $J=\sigma E$.

A box with $i \times j \times k=72 \times 42 \times 234$ mesh cells was used to cover the human body. The maximum eddy current density along the human body ( $Z$-direction) is shown in Figure 5a. Details of eddy current density in $X-Z$ cross section are given in Figure 5b.

This study highlights that the HR-FDTD method is capable of solving high resolution problems in complex situations. The physical time required for a stable solution is only about $0.3 \%$ of the source 


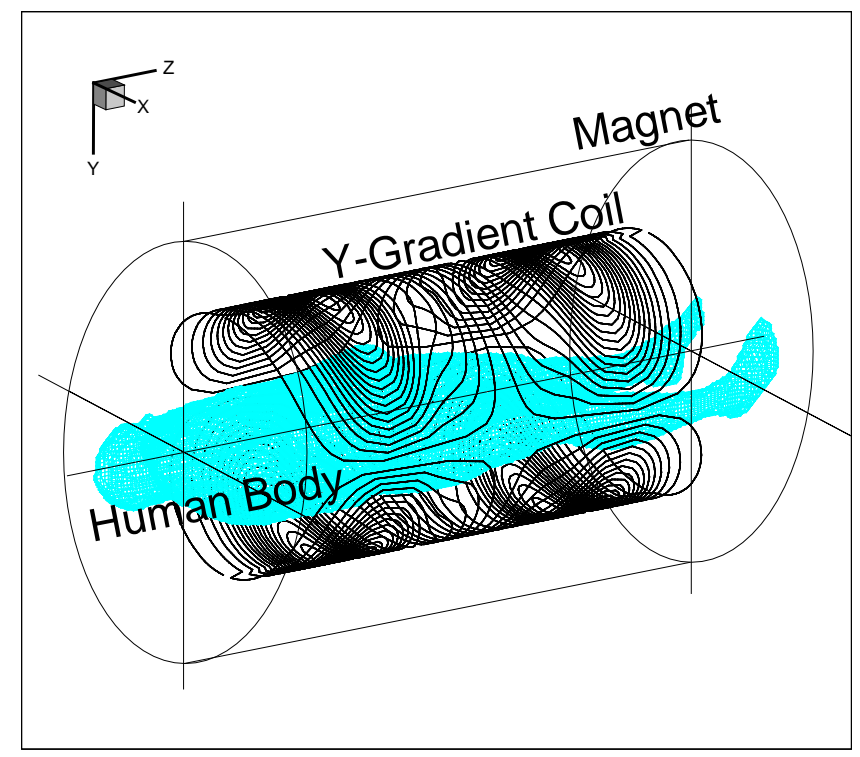

FiguRE 4: An outline of the body model inside an MRI system and surrounded by a $Y$-gradient coil.

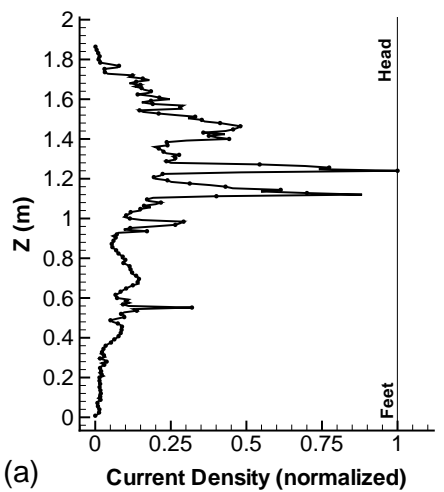

(b)

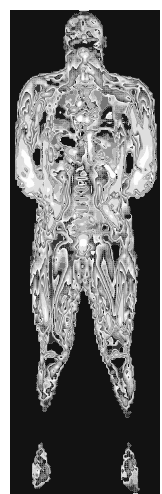

Figure 5: (a) The maximum eddy current density along the human body ( $Z$-direction) produced by a $40 \mathrm{mT} / \mathrm{m} Y$-gradient oscillating at $833 \mathrm{~Hz}$. (b) A coronal section of the complete solution with white is high density and dark is lower density. 
period. The accuracy and the efficiency of the model are satisfactory for further investigation of biological effects in MRI systems. These results were obtained after approximately $24 \mathrm{hrs}$ of computation on a SUN Enterprise 450.

\section{Conclusion}

A new high resolution FDTD method has been described. The method is essentially frequency independent, having been tested over an extended frequency range $(50 \mathrm{~Hz}-1 \mathrm{GHz})$. As an example of a bioelectromagnetic application, the HR-FDTD method has been used to compute eddy-current induction in a human body model due to gradient coil pulsing in an MRI system.

It is hoped that further studies, which include more complex source functions, will be presented in the near future.

\section{References}

[1] J. P. Berenger. A perfectly matched layer for the absorption of electromagnetic waves. Journal of Computational Physics, 114, 185-200, 1994. C859

[2] C. M. Furse and O. P. Gandhi. Calculation of electric fields and currents induced in a millimeter-resolution human model at $60 \mathrm{~Hz}$ using the FDTD method. Bioelectromagnetics, 19,293-299, 1998. C853, C855 
[3] R. Holland. Finite-difference time-domain (FDTD) analysis of magnetic diffusion. IEEE Trans. Electromagnetic Compatibility, 36, 1, 32-39, 1994. C854, C855

[4] W. L. Ko and R. Mittra. Extremely low frequency modeling in lossy media using FDTD with application in seafloor characterization. Electromagnetics, 15: 587-602,1995.

[5] J. D. Moerloose, T. W. Dawson, and M. A. Stuchly. Application of the finite difference time domain algorithm to a quasi-static field analysis. Radio Science, 32(2), 329-341,1997. C854, C855, C857

[6] W. C. Parkinson. Electromagnetic fields in biological studies. Annals of Biomedical Engineering, 3, 491-514, 1985. C853

[7] J. P. Reilly. Peripheral nerve stimulation by induced electric currents: exposure to time-varying magnetic fields. Medical and Biological Engineering and Computing, 27, 101-110, 1989. C853, C864

[8] K. Umashankar and A. Taflove. A novel method to analyze electromagnetic scattering of complex objects. IEEE Trans. Electromagnetic Compatibility, 24, 397-405, 1982. C854

[9] K. S. Yee. Numerical solution of initial boundary value problems involving Maxwell's equations in isotropic media. IEEE Trans. Antennas Propagat., 14, 302-307, 1966. C854 